\title{
Triple therapy with salmeterol/fluticasone propionate $50 / 250$ plus tiotropium bromide improve lung function versus individual treatments in moderate-to- severe Japanese COPD patients: a randomized controlled trial - Evaluation of Airway sGaw after treatment with tripLE [Corrigendum]
}

Saito T, Takeda A, Hashimoto K, et al. Int J Chron Obstruct Pulmon Dis. 2015;10:2393-2404.

On page 2395, right column, line 3, "Philadelphia, PA, USA" should have read "Hoechberg, Germany".

On page 2396, Figure 2 was incorrect. The corrected figure is shown below.

On page 2396, right column, line 1, "although $\mathrm{FEV}_{1}$ and FVC were not log transformed prior to analysis" should have read "although sRaw and sGaw were log transformed prior to analysis".

On page 2397, right column, Lung volumes section, line 5, "Statistical significance was observed for postdose RV immediately after dosing (time $=0$ ) on day 28 for SFC250 + TIO compared to TIO and SFC250 (Tables S3, S4). Improvements on day 28 were also seen in postdose TGV, IC, and TLC following treatment with SFC250 + TIO compared to each component, but none were significant (Table S4). Improvements were seen for all parameters for their adjusted

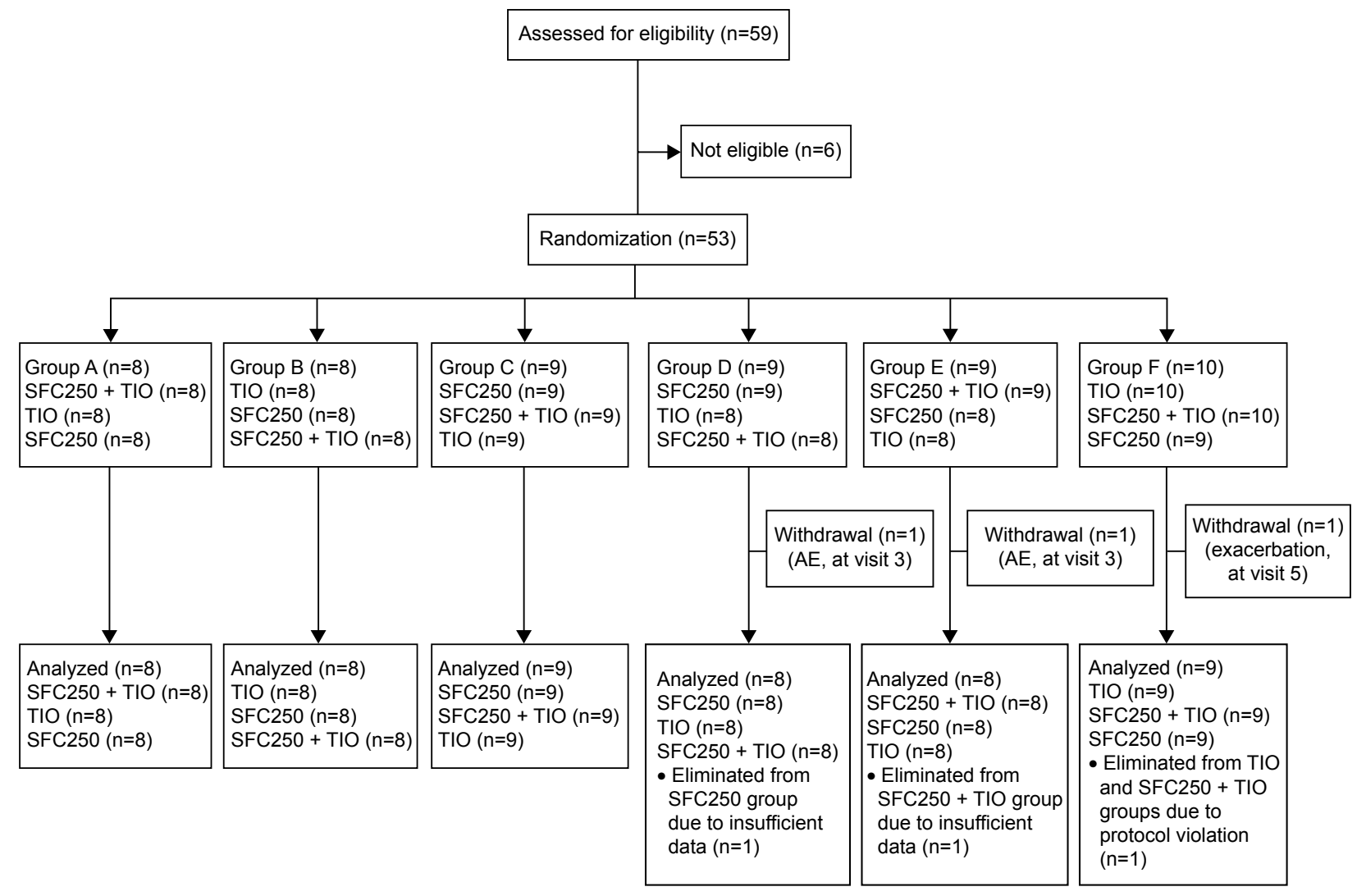

Figure 2 CONSORT flow diagram.

Abbreviations: AE, adverse event; SFC, salmeterol/fluticasone propionate; sGaw, specific airway conductance; TIO, tiotropium bromide. 
mean values on day 1 , but none were significant (Table S3)." Should have read "Statistical significance was observed for trough RV (time $=0$ ) on day 28 for SFC250 + TIO compared to TIO and SFC250 (Table S3). Improvements on day 28 were also seen in postdose TGV, IC, and TLC following treatment with SFC250 + TIO compared to each component, but statistical treatment differences were not observed, except for after dosing (240 $\mathrm{min}$ ) IC at day 28 (Table S3). Improvements were seen for all parameters for their adjusted mean values on day 1 , but statistical treatment differences were not observed, except for SFC250 after dosing (240 min) TGV at day 1 (Table S3)."

On page 2398, Figure 3 was incorrect. The corrected figure is shown below.

On page 2398, Table 3, TGV (L) row, SFC250 + TIO vs TIO $(n=50)$ : $97.5 \%$ CI column, “-0.029” should have read " $0.029 ”$.

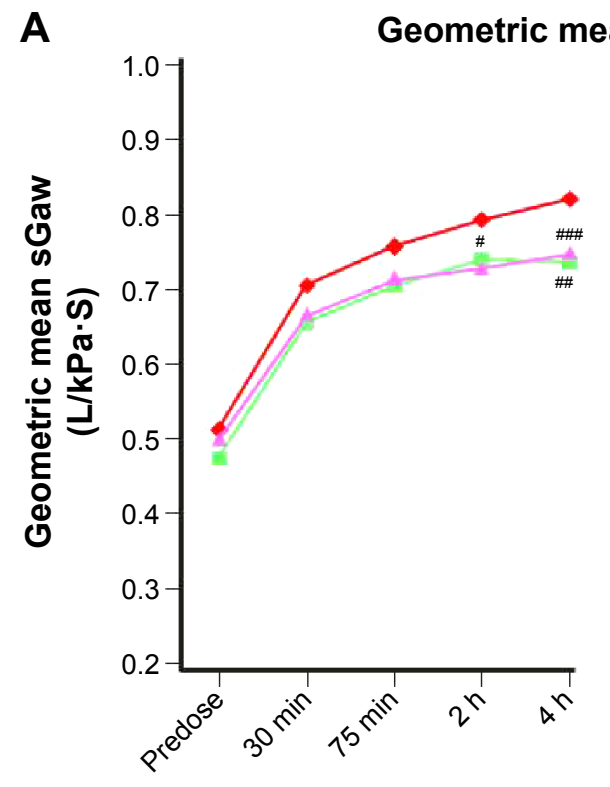

Day 1

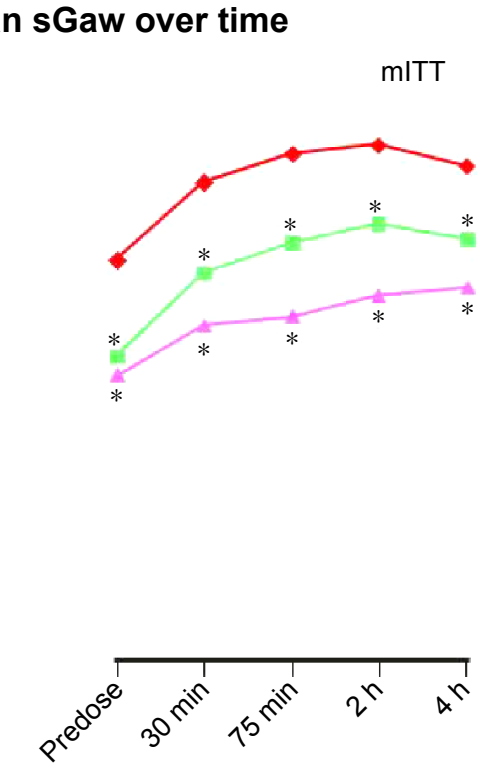

Day 28

\begin{tabular}{|c|c|c|c|}
\hline \multirow[t]{2}{*}{ Symbol } & $\leadsto$ Triple & -E-1il TIO & \\
\hline & $\begin{array}{l}\text { SFC: }{ }^{\#} P=0.019 \\
\text { TIO: }{ }^{\# \#} P=0.004\end{array}$ & $\# P=0.007$ & $* P<0.001$ \\
\hline
\end{tabular}
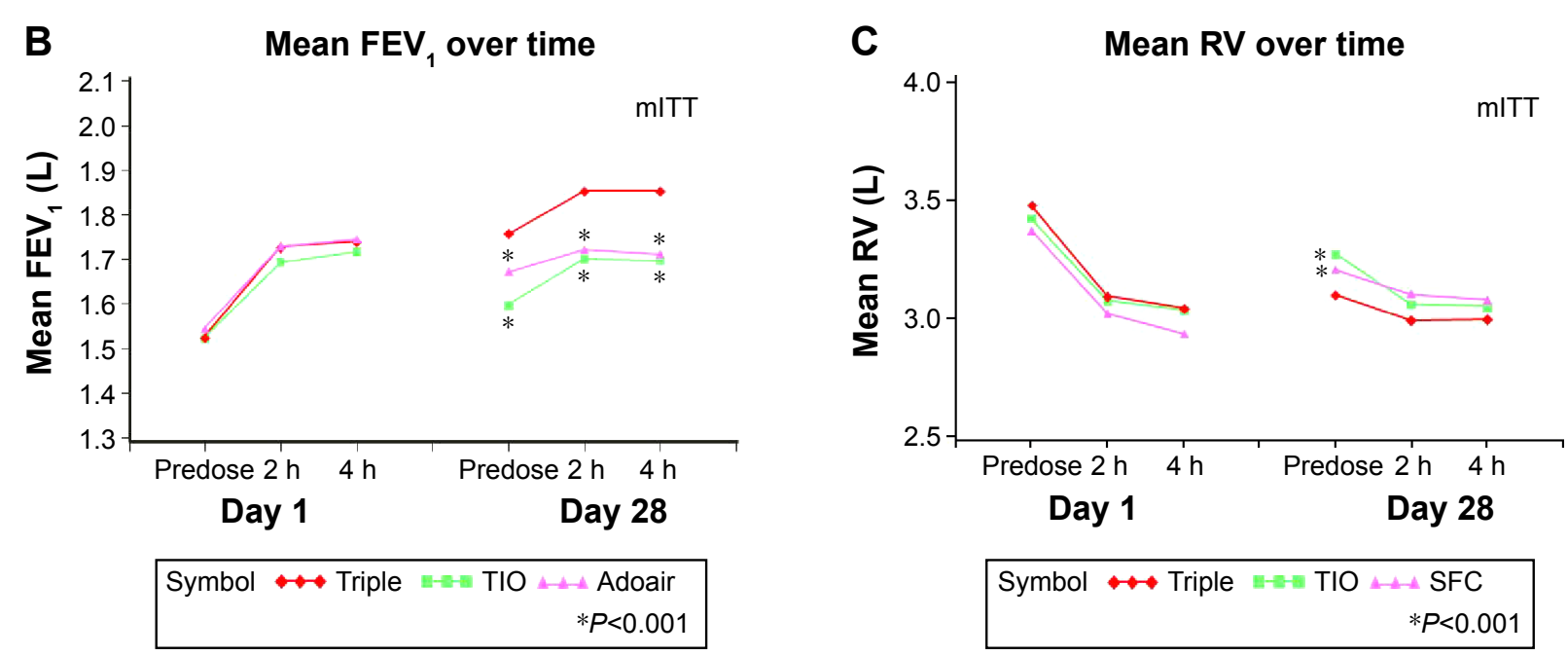

Figure 3 (A) sGaw, (B) $\mathrm{FEV}_{1}$, and (C) RV on days I and 28 of treatment.

Abbreviations: mITT, modified intent-to-treat; $\mathrm{FEV}_{1}$, forced expiratory volume in I second; sGaw, specific airways conductance; RV, residual volume; SFC, salmeterol/ fluticasone propionate combination; TIO, tiotropium bromide; min, minutes; h, hours. 
On page 2398, Table 3, TGV (L) row, SFC250 + TIO vs SFC250 (n=50): 97.5\% CI column, “-0.028” should have read " 0.028 ".

On page 2398, Table 3, Note section, "Difference" should have read "Adjusted mean difference".

On page 2402, Table S2, figure caption, "Postdose raw mean (standard deviation $\log$ ) values of sGaw and sRaw (mITT population)" should have read "Postdose raw geometric mean (standard deviation log) values of sGaw and sRaw (mITT population)".

On page 2402, Table S2, Time (minutes) column, sGaw, third line down, "SFC250 + TIO" should have read "SFC250".

On page 2402, Table S2, Time (minutes) column, sRaw, third line down, "SFC250 + TIO" should have read "SFC250".

\section{Publish your work in this journal}

The International Journal of COPD is an international, peer-reviewed journal of therapeutics and pharmacology focusing on concise rapid reporting of clinical studies and reviews in COPD. Special focus is given to the pathophysiological processes underlying the disease, intervention programs, patient focused education, and self management protocols.

\section{Dovepress}

This journal is indexed on PubMed Central, MedLine and CAS. The manuscript management system is completely online and includes a very quick and fair peer-review system, which is all easy to use. Visit http://www.dovepress.com/testimonials.php to read real quotes from published authors. 